\title{
The Effect of Basis Selection on Thermal-Acoustic Random Response Prediction Using Nonlinear Modal Simulation
}

\author{
Stephen A. Rizzi ${ }^{*}$ \\ NASA Langley Research Center, Structural Acoustics Branch, Hampton, VA 23681 \\ Adam Przekop \\ National Institute of Aerospace, Hampton, VA 23666
}

\begin{abstract}
The goal of this investigation is to further develop nonlinear modal numerical simulation methods for prediction of geometrically nonlinear response due to combined thermalacoustic loadings. As with any such method, the accuracy of the solution is dictated by the selection of the modal basis, through which the nonlinear modal stiffness is determined. In this study, a suite of available bases are considered including (i) bending modes only; (ii) coupled bending and companion modes; (iii) uncoupled bending and companion modes; and (iv) bending and membrane modes. Comparison of these solutions with numerical simulation in physical degrees-of-freedom indicates that inclusion of any membrane mode variants (ii - iv) in the basis affects the bending displacement and stress response predictions. The most significant effect is on the membrane displacement, where it is shown that only the type (iv) basis accurately predicts its behavior. Results are presented for beam and plate structures in the thermally pre-buckled regime.
\end{abstract}

\section{Nomenclature}

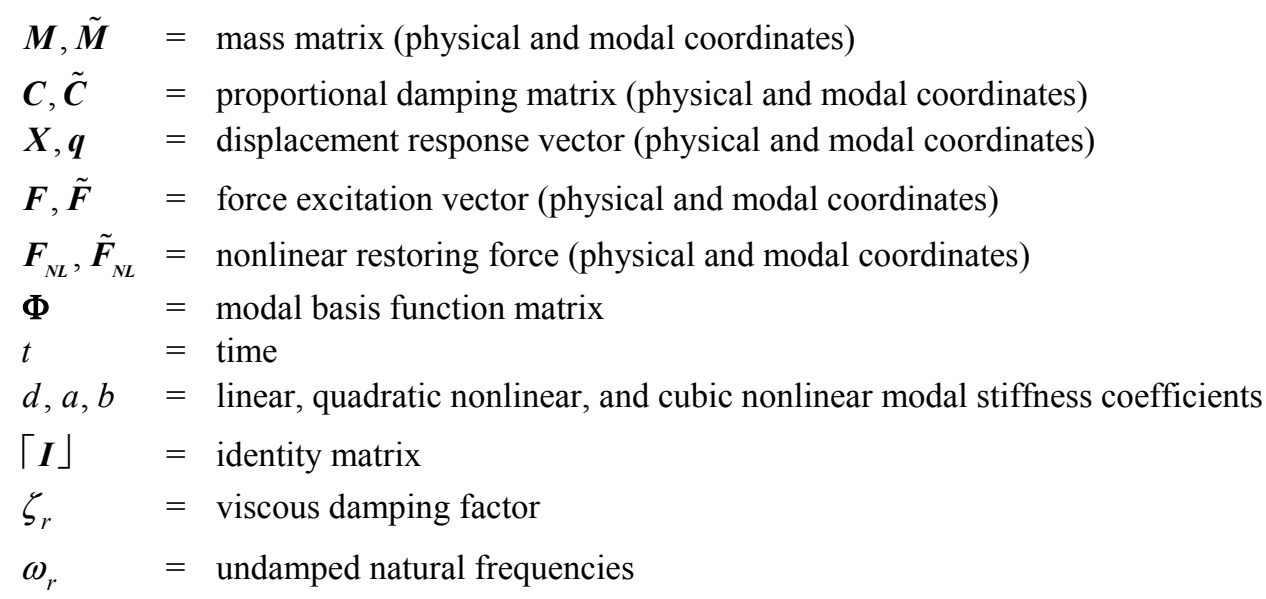

\section{Introduction}

Future aircraft and launch vehicle structures will be exposed to increasing thermal and acoustic loads. In order to avoid the premature failure due to high cycle fatigue, fast and accurate analysis methods are required for use in the design process. The goal of this investigation is to further develop finite-element-based nonlinear modal numerical simulation methods for prediction of geometrically nonlinear response due to combined thermal-acoustic loadings. These developments will ultimately form the basis of a computationally efficient thermal-acoustic fatigue design tool.

\footnotetext{
*Aerospace Engineer, AIAA Associate Fellow

${ }^{\dagger}$ Staff Scientist, AIAA Member
} 
Reduced order analysis methods gain their computational advantage over direct numerical simulation by transforming the equations of motion in physical degrees-of-freedom to modal degrees-of-freedom. Hence system size may be significantly reduced. In the recent past, a significant amount of research has been performed in the area of reduced order methods development. These may be viewed as being in one of two categories; those in which the nonlinear modal stiffness is directly evaluated from the nonlinear finite element stiffness matrix (so-called direct methods), and those in which the nonlinear modal stiffness is indirectly evaluated. Direct methods are typically implemented in special purpose finite element codes in which the nonlinear stiffness is known. The work by Mei et $\mathrm{al}^{1}$ for flat configurations and the expansion of that approach for curved panels by Przekop et $\mathrm{al}^{2,3}$ are good examples of the direct method. Indirect stiffness evaluation methods are typically implemented for use with commercial finite element codes in which the nonlinear stiffness is unavailable. Examples of indirect stiffness evaluation approaches may be found in the work of McEwan et $\mathrm{al}^{4}$ and Muravyov and Rizzi. ${ }^{5}$ The latter implementation, as embodied in the code ELSTEP ${ }^{6}$, forms the basis for the current work. ELSTEP uses MSC.NASTRAN 2001 to evaluate the nonlinear stiffness.

For both direct and indirect stiffness evaluation approaches, the crux of the problem lies in the selection of the proper basis, through which the nonlinear modal stiffness may be determined. If an insufficient basis is selected, then the predicted dynamic response of the reduced order model may significantly differ from that of the full nonlinear model. Thus, there is a need in all circumstances to assess the basis used through comparison of the predicted response with something other than a reduced order method, e.g. experimental data or numerical simulation in physical degrees-of-freedom. Recent work by Hollkamp et al ${ }^{7}$ compared various reduced order approaches with experimental data. The use of experimental data, however, may be difficult, as both thermal and mechanical boundary conditions must be matched between the reduced order finite element analysis and experiment. Numerical simulation in physical degrees-of-freedom eliminates this difficulty as thermal and mechanical boundary conditions may be identically specified, though at the cost of a computationally intensive analysis. This approach has been successfully employed to validate a reduced order equivalent linearization analysis in the absence of thermal loading. ${ }^{8}$

The objective of the present work is to assess the effect of basis selection on thermal-acoustic random response prediction in the pre-buckled regime using a nonlinear modal simulation. For this class of problems, geometric nonlinearity couples the bending and membrane response, indicating a need to represent the effect of both in the basis selection. In an effort to determine which selection is most accurate, a suite of bases are considered including (i) bending modes only; (ii) coupled bending and companion modes; (iii) uncoupled bending and companion modes; and (iv) bending and membrane modes. Results from nonlinear modal simulation are compared with results obtained through numerical simulation in physical coordinates. Planar aluminum beam and plate structures are considered.

\section{Nonlinear Modal Simulation}

The nonlinear modal simulation analysis consists of several parts. The linear system matrices are first obtained from a commercial finite-element program. One or more methods, to be discussed, are next used to obtain a modal basis. Following a transformation of the nonlinear system to modal coordinates, the modal stiffness coefficients are evaluated and the resulting coupled system of equations is numerically integrated to obtain the modal displacement time history. These are transformed back to physical coordinates for post-processing, including stress recovery.

\section{A. Modal Coordinate Transformation}

The equations of motion of the nonlinear system in physical degrees-of-freedom may be written as

$$
\boldsymbol{M} \ddot{\boldsymbol{X}}(t)+\boldsymbol{C} \dot{\boldsymbol{X}}(t)+\boldsymbol{F}_{N L}(\boldsymbol{X}(t))=\boldsymbol{F}(t) .
$$

A set of coupled modal equations with reduced degrees-of-freedom is first obtained by applying the modal coordinate transformation $\boldsymbol{X}=\boldsymbol{\Phi} \boldsymbol{q}$ to Eq. (1). The modal basis function matrix $\boldsymbol{\Phi}$ is typically formed from the eigenvectors obtained from Eq. (1) using only the linear stiffness. For flat isotropic structures, these may include any combination of bending and membrane modes. In lieu of membrane modes, the modal basis may include "companions" related to the membrane response, as discussed in the next section. Generally, a small set of $L$ basis functions are included giving

$$
\tilde{\boldsymbol{M}} \ddot{\boldsymbol{q}}+\tilde{\boldsymbol{C}} \dot{\boldsymbol{q}}+\tilde{\boldsymbol{F}}_{N L}\left(q_{1}, q_{2}, \ldots, q_{L}\right)=\tilde{\boldsymbol{F}}
$$


The time dependence of $q, \tilde{\boldsymbol{F}}$ and $\tilde{\boldsymbol{F}}_{N L}$ is implied, and

$$
\tilde{\boldsymbol{M}}=\boldsymbol{\Phi}^{T} \boldsymbol{M} \boldsymbol{\Phi}=\lceil\boldsymbol{I}\rfloor \quad \tilde{\boldsymbol{C}}=\boldsymbol{\Phi}^{T} \boldsymbol{C} \boldsymbol{\Phi}=\left\lceil 2 \zeta_{r} \omega_{r}\right\rfloor \quad \tilde{\boldsymbol{F}}_{N L}=\boldsymbol{\Phi}^{T} \boldsymbol{F}_{N L} \quad \tilde{\boldsymbol{F}}=\boldsymbol{\Phi}^{T} \boldsymbol{F} .
$$

\section{B. Modal Basis Calculation}

For the problems of interest in this paper, both bending and membrane behavior should be included in the basis selection since the large deflection nonlinearity couples their response. The basis functions may be determined via several methods. Basis functions corresponding to the bending and membrane response may be determined through solution of the linear eigenvalue problem. Other basis functions corresponding to the membrane response induced by bending-membrane coupling may be determined via alternative approaches. Lastly, while not the subject of this paper, basis functions may also be determined via experiment or hybrid scheme.

\section{Linear Eigenvectors}

In this study, only isotropic materials or laminated composite materials having zero laminate coupling stiffness $([\mathrm{B}]=0)$ are considered. Further, the current work is restricted to planar structures, i.e. flat beams and plates. The linear eigenvectors obtained from Eq. (1) (using only the linear stiffness) are therefore uncoupled and are either associated with low-frequency bending modes or high-frequency membrane modes. The selection of which bending modes to include depends on both the excitation bandwidth and the loading distribution. The identification of each selected bending mode is straightforward as they are typically the lowest frequency modes. The selection of which membrane modes to include is less apparent. All or most membrane modes will likely fall outside the excitation bandwidth. Furthermore, the identification of any particular membrane mode may be difficult and time consuming. Nevertheless, a reasonable starting point is to select the lowest membrane modes that are consistent with the loading distribution. Inclusion of both these eigenvectors in the modal basis, either independently or in pairs, is subsequently referred to as the bending and membrane mode (BM) case. Inclusion of only the bending eigenvectors will be referred to as the bending mode only (B) case.

In this study, the mass-normalized linear eigenvectors without the effect of thermal stress were obtained using MSC.NASTRAN normal modes analysis (solution 103). These are the so-called "cold" modes. The massnormalized linear eigenvectors with the effect of thermal stress, i.e. "hot" modes, requires MSC.NASTRAN nonlinear static analysis (solution 106).

\section{Companion Basis Functions}

An alternative approach to using membrane modes is the use of so-called companion ${ }^{7}$ (or dual ${ }^{9}$ ) modes. These modes represent the membrane behavior resulting from bending due to bending-membrane coupling. Previous authors utilized quasi-static approaches to determine the companion mode via nonlinear static analyses. For each companion mode, Hollkamp et $\mathrm{al}^{7}$ prescribed a displacement field corresponding to the bending mode of interest. Mignolet et $\mathrm{al}^{9}$ applied two loadings, each having the distribution of a particular bending mode shape but with different magnitudes, to obtain the companion for that bending mode. In either case, the resulting modes contained only the membrane related behavior, but not the bending.

A new method is now presented for computing the companion mode using a dynamic analysis. An initial stressfree imperfection is first introduced in the flat structure to couple the bending-membrane response. The magnitude of the imperfection is chosen to be very small such that a normal modes analysis yields virtually the same bending eigenvalues and eigenvectors as that of the flat structure. An engineering rationale for specifying the shape of the imperfection is not evident. In this paper, the shape was chosen to be that of the first bending mode. It was found, however, that other imperfection shapes resulted in different companion parts of the eigenvectors. The bending and high frequency membrane components remained identical to that of the flat structure regardless of the shape of the imperfection. For the cold modes, MSC.NASTRAN solution 103 was again used to compute the mass-normalized eigenvectors, which now contain both the bending and membrane behaviors, but at the natural frequencies of the original flat structure. Direct inclusion of these eigenvectors in the modal basis is subsequently referred to as the coupled bending and companion mode (CBC) case. A more consistent usage, however, is to separate the degreesof-freedom (DoFs) associated with the bending and membrane behaviors. In practice, since the bending behavior is unchanged, the original low-frequency bending modes are retained. The bending DoFs are set to zero in the newly obtained eigenvector to obtain the dynamic companion. Inclusion of both these eigenvectors in the modal basis, independently or in pairs, is subsequently referred to as the uncoupled bending and companion mode (UBC) case. 


\section{Comparison of Membrane and Companion Modes}

Consider a clamped-clamped aluminum beam measuring 18-in. $\times 1$-in. $\times 0.09$-in $(l \times w \times h)$ with the following material properties:

$$
E=10.6 \times 10^{6} \text { psi, } \quad G=4.0 \times 10^{6} p s i, \quad \rho=2.588 \times 10^{-4} \frac{l b_{f}-s^{2}}{i n^{4}} .
$$

The beam was modeled using seventy-two CBEAM elements. The first four symmetric bending modes (eigenvectors 1, 3, 7 and 10) are at natural frequencies of 58,312, 770 and $1430 \mathrm{~Hz}$, respectively. The first four anti-symmetric membrane modes (eigenvectors $47,86,127,140$ ) are at natural frequencies of 11.2, 22.5, 33.6 and $44.8 \mathrm{kHz}$, respectively. These are plotted in Figure 1 - Figure 4. Also shown are the static companion modes obtained by Mignolet's approach ${ }^{9}$ and the dynamic companion modes obtained by the method outlined above. It is clear that both static and dynamic companion modes significantly differ in shape relative to the membrane mode. The effect of their inclusion in the modal basis on the stiffness coefficients is next considered.

\section{Indirect Stiffness Evaluation Method}

The indirect stiffness evaluation method previously developed without the effect of thermal loading ${ }^{5}$ was further developed for thermal loadings. ${ }^{9}$ To summarize, the nonlinear force vector in Eq. (2) may be written in the form

$$
\tilde{F}_{N L}\left(q_{1}, q_{2}, \ldots, q_{L}\right)=\sum_{j=1}^{L} d_{j}^{r} q_{j}+\sum_{j=1}^{L} \sum_{k=j}^{L} a_{j k}^{r} q_{j} q_{k}+\sum_{j=1}^{L} \sum_{k=j}^{L} \sum_{l=k}^{L} b_{j k l}^{r} q_{j} q_{k} q_{l} \quad r=1,2, \ldots, L
$$

reducing the problem of determining the nonlinear stiffness from one in which a large set of simultaneous nonlinear equations must be solved to one involving simple algebraic relations. For the prescribed static displacement fields ${ }^{9}$

$$
\boldsymbol{X}_{c 1}=+\phi_{1} q_{1} \quad \boldsymbol{X}_{c 2}=-\phi_{1} q_{1} \quad \boldsymbol{X}_{c 3}=+\phi_{1} \hat{q}_{1}
$$

the nonlinear forces evaluated using the MSC.NASTRAN nonlinear static solution (solution 106) are given as

$$
\begin{aligned}
& \tilde{\mathbf{F}}_{N L_{1}}=\boldsymbol{\Phi}^{T} \mathbf{F}_{N L}\left(+\phi_{1} q_{1}\right)=+\left[d_{1}^{r}\right] q_{1}+\left[a_{11}^{r}\right] q_{1} q_{1}+\left[b_{111}^{r}\right] q_{1} q_{1} q_{1} \\
& \tilde{\mathbf{F}}_{N L_{2}}=\boldsymbol{\Phi}^{T} \mathbf{F}_{N L}\left(-\phi_{1} q_{1}\right)=-\left[d_{1}^{r}\right] q_{1}+\left[a_{11}^{r}\right] q_{1} q_{1}-\left[b_{111}^{r}\right] q_{1} q_{1} q_{1} . \\
& \tilde{\mathbf{F}}_{N L_{3}}=\boldsymbol{\Phi}^{T} \mathbf{F}_{N L}\left(+\phi_{1} \hat{q}_{1}\right)=+\left[d_{1}^{r}\right] \hat{q}_{1}+\left[a_{11}^{r}\right] \hat{q}_{1} \hat{q}_{1}-\left[b_{111}^{r}\right] \hat{q}_{1} \hat{q}_{1} \hat{q}_{1}
\end{aligned}
$$

The first two of Eqs. (7) are used to evaluate $\left[a_{11}^{r}\right]$. The first and the third of Eqs. (7) are then used to determine $\left[d_{1}^{r}\right]$ and $\left[b_{111}^{r}\right]$. It should be noted that the specified modal displacements in Eq. (6) are single scalar quantities since the analysis is static. These modal displacements are specified such that the magnitude of the prescribed physical displacement field $\boldsymbol{X}_{c}$ is physically meaningful. It has been shown that the stiffness coefficients obtained via this approach are not sensitive to the particular value of modal displacement specified. ${ }^{5}$ The last of Eqs. (6) are obtained by specifying a scaled modal displacement such that $\hat{q}=\alpha q$. In this paper, a value of $\alpha=1.25$ is used.

This procedure for evaluating the linear stiffness coefficients $\left[d_{1}^{r}\right]$ is advantageous over the earlier implementation ${ }^{5}$ using the MSC.NASTRAN linear static solution (solution 101), as the linear solution does not include in-plane displacements, and therefore is not capable of handling thermal loads. The remaining nonlinear coefficients are evaluated through an additional series of nonlinear static solutions. ${ }^{5}$

\section{Comparison of Modal Stiffness Coefficients}

For the clamped-clamped beam structure previously considered, the effect of including a particular set of modes in the modal basis is shown in Table $1-3$. Table 1 shows the diagonal terms of the linear stiffness coefficients for a set consisting of the first symmetric bending modes $\left(d_{1}^{1}-d_{4}^{4}\right)$ plus up to four membrane or companion modes $\left(d_{5}^{5}\right.$ $\left.d_{8}^{8}\right)$. In each case, the first four linear stiffness coefficients are equal to the eigenvalues of the selected bending 
modes. For the BM case, the second four linear stiffness coefficients correspond to the eigenvalues of the selected membrane modes. The CBC case lacks the second set of four coefficients as only four modes are included in this basis, i.e. four coupled bending and companion modes. Both versions of the UBC case indicate low frequency behavior for the second set of four (companion) modes. In all cases but $\mathrm{CBC}$, the off-diagonal terms are effectively zero (not shown).

Since the beam structure is flat, all quadratic stiffness coefficients are nominally zero and are not shown. Table 2 shows the diagonal terms of the cubic stiffness coefficients for the same modal bases as above. In each case, all coefficients corresponding to the bending modes (1-4) are identical, indicating that the effect of the different modal bases is reflected in the off-diagonal terms that couple the various modes. This is more apparent in Table 3 , which shows selected off-diagonal stiffness coefficients corresponding to the first $\left(1^{\text {st }}\right.$ symmetric bending) and fifth $\left(1^{\text {st }}\right.$ membrane or companion) modes. There is a much greater coupling of bending and membrane modes in the BM basis than any of the other bases, as shown by stiffness terms $b_{155}^{1}$ and $b_{115}^{5}$. Additionally, the relationship between non-zero stiffness terms, ${ }^{5}$

$$
b_{155}^{1}=b_{115}^{5}
$$

is approximately maintained for the BM and UBC bases, and somewhat less so for these particular terms in the static UBC basis. Further discussion regarding the choice of basis on the response prediction is reserved until Sec. III.

\section{Numerical Integration and Element Stress Recovery}

Having the nonlinear force vector in Eq. (5) fully defined, the coupled modal nonlinear equations of motion in Eq. (2) are numerically integrated using a fourth-order Runge-Kutta method. ${ }^{10}$ The resulting modal displacement time histories are transformed back to physical coordinates using the inverse modal transformation. A fixed time integration step of between $50 \mu s$ (for the lowest excitation levels) and $10 \mu s$ (for the highest excitation levels) was used.

In contrast to mapping techniques relating stress to physical DoFs, ${ }^{7,9}$ the approach taken here recovers the element stresses by post-processing the nodal physical DoFs directly within the finite element program. Since the stress post-processor of the finite element program is used, the stresses are identical to those that would have been obtained via a standard finite element analysis in physical DoFs. The appeal of direct stress recovery over mapping techniques is that it eliminates any issues concerning the accuracy of the mapping, and eliminates the need for estimating nonlinear mapping functions for each stress quantity of interest. The disadvantage is that it can be computationally costly.

In principal, the approach taken is simple. For a single element stress recovery, a finite element mesh is made consisting of two elements. The stress recovery element is of the same type and has the same properties of the element of interest. For a particular output time step, the element physical DoFs, obtained via the nonlinear modal simulation method, are applied to each element node as prescribed displacement fields in the MSC.NASTRAN nonlinear static solution. A nuance of MSC.NASTRAN requires the attachment of a dummy element and load ${ }^{6}$ to the stress recovery element, in order to calculate the resulting element nodal forces and recover the element stress. By repeating this operation for each output time step, the stress time history is determined. If stress recovery is required for a region or the entire structure, that portion of the original model is used and a dummy element is attached. The prescribed displacement field spans the entire region of interest.

\section{E. Convergence}

There are several aspects relating to eigenvalue and modal convergence that warrant discussion. A means of assessing the adequacy of the finite element mesh within a given frequency range is to check convergence of the eigenvalues. In the analyses performed herein, eigenvalue convergence was checked through the applied loading bandwidth. For the beam, natural frequencies of the membrane modes in the BM basis $(11.2-44.8 \mathrm{kHz})$ were significantly higher than the loading bandwidth of $0-1500 \mathrm{~Hz}$. Further, it is known that the membrane response has double the frequency content of the loading due to the fact that the membrane vibration undergoes two cycles for every one cycle of bending. In this study, no attempt was made to further refine the finite element mesh to achieve eigenvalue convergence to either double the maximum loading frequency or to the membrane mode eigenvalue. The implication of the latter is that membrane eigenvectors included in the basis will be less accurate than the bending eigenvectors.

For any given modal basis variant, modal convergence (the effect of adding additional modes to the basis) must be assessed through convergence of the predicted response in either modal or physical coordinates. Since the 
stiffness coefficients are independent of each other, the inclusion of additional modes in the basis only augments the size of the modal stiffness matrices. The addition of the $n^{\text {th }}$ basis does not affect the magnitudes of the coefficients evaluated using $n-l$ basis functions.

\section{Displacement and Stress Response Results}

\section{A. Numerical Simulation in Physical Coordinates}

Numerical simulation analysis in physical coordinates served as the basis for comparison of results from nonlinear modal simulation analyses. The finite element program ABAQUS (version 6.4) was to generate nonlinear displacement and stress time histories. The double precision explicit integration scheme with an adaptive time integration step (referred to as "element by element" in ABAQUS) was utilized for all analyses. The mass proportional damping factor used was the same as that specified in the nonlinear modal simulation analyses. The choice of ABAQUS over alternative analysis methods, e.g. MSC.NASTRAN nonlinear transient solution (solution 129), was made based on its superior ability to simulate very long response histories. This choice introduced an inconsistency of element formulations used between the nonlinear modal and physical simulations. However, nearly identical nonlinear static analysis results from MSC.NASTRAN and ABAQUS (not shown) established the consistency of displacement and stress.

\section{B. Load Generation, Stress Post-Processing, and Ensemble Averaging}

The same loading time history, ensemble averaging, and stress post-processing was used for the numerical simulation analysis in physical and modal coordinates. The loading time histories were generated by summing equal amplitude sine waves, each with random phase, within the specified bandwidth using a discrete inverse Fourier transform. This procedure was identical to that used in previous work ${ }^{8}$ by the authors, so further details are omitted for brevity. The loading produced by this method has a Gaussian distribution. A sharp roll-off of the input spectrum practically eliminates excitation of the structure outside the frequency range of interest.

The longitudinal stresses at the upper and lower surfaces were computed using the method previously described for the nonlinear modal simulation, or directly by ABAQUS for the simulation in physical coordinates. The bending and membrane components were separated by averaging the upper and lower surface stresses to obtain the membrane component, and then subtracting that from the surface stress to obtain the bending component. This separation allowed greater insight into the effect of the various modal bases on the stress response.

A range of load levels was considered to span the response regime from nearly linear to highly nonlinear. For each load level, ten ensembles of displacement and stress response were generated lasting $2.1384 \mathrm{~s}$ each. The first $0.5 \mathrm{~s}$ of each response record was discarded to remove the initial transient response, ${ }^{8}$ resulting in response histories of $1.6384 \mathrm{~s}$ in duration. In the case of the nonlinear modal simulation, the transient response was removed from the displacement time history prior to stress recovery. For each simulation, the displacement and stress response was stored at every $50 \mu \mathrm{s}$, giving time records of 32,768 points. A 32,768-point FFT was subsequently used to compute the power spectral density (PSD) function.

\section{Beam Results}

The clamped-clamped beam structure previously discussed was first considered. The MSC.NASTRAN model consisted of seventy-two CBEAM elements. Nonlinear modal simulations were run using four of the five modal bases from Table 1-3; B, BM, CBC and UBC. Simulations for the fifth basis, UBC static, were run for a subset of conditions. The ABAQUS model consisted of seventy-two B21 elements. Damping was chosen to be sufficiently high so that a good comparison could be made at the peaks of the PSD. A level of mass proportional damping was specified corresponding to critical damping of $2.0 \%$ for the first symmetric bending mode. The beam was subjected to a uniformly distributed transverse loading with a bandwidth of $1500 \mathrm{~Hz}$. Two thermal conditions were investigated; a room temperature condition and an elevated temperature $\left(\Delta T=5^{\circ} \mathrm{F}\right)$ below the critical thermal buckling temperature $\left(\Delta T_{C R}=6.64^{\circ} \mathrm{F}\right)$.

Displacement results are presented at the $23 \%$ span (4.125-in. from the clamped end), and element stresses at $21 \%$ span (3.75-in. from the clamped end). These locations help to elucidate the benefits and liabilities of the various modal bases under severe conditions as the membrane stress component is more significant there relative to the bending stress component than at other locations along the length, e.g. the clamped end or mid-span. 


\section{Room Temperature}

The root-mean-square (RMS) transverse displacement is shown in Figure 5 for a range of loadings. Nonlinearity is seen to increase with increasing load. The best comparison with numerical simulation in physical DoFs is achieved using the BM basis. Both UBC and $\mathrm{CBC}$ bases are seen to offer some improvement relative to the bending only basis. The RMS membrane displacement is shown in Figure 6. Compared to the ABAQUS results, the level of agreement achieved using the BM basis is excellent. The largest contribution to the RMS membrane displacement using the UBC comes from the low frequencies of the companion modes (see Table 1). This will be further substantiated by the membrane displacement PSDs to follow. In an RMS sense, the membrane displacements obtained using the $\mathrm{CBC}$ basis were negligible. Note that the bending only basis is incapable of generating any membrane displacement.

The transverse displacement PSDs are shown in Figure 7 for the lowest loading of $7.2 \times 10^{-3} \mathrm{lb} / \mathrm{in}$. For this level, all modal bases give essentially the same results as the numerical simulation in physical DoFs. The membrane displacement PSDs for this load level, shown in Figure 8, offer a different perspective. The BM basis is the only one to compare well with the ABAQUS results. The CBC basis is inaccurate on two accounts; the magnitude is clearly incorrect and the shape of the frequency response mimics that of the bending response in Figure 7. It comes as no surprise that both UBC bases compare poorly. The magnitude of bending-companion coupling in the cubic stiffness coefficients are orders of magnitude lower than the bending-membrane coupling in the BM basis, see Table 3 , and therefore account for the near negligible displacement response other than at very low frequency. Since the two UBC bases give very similar results, differences between the stiffness terms in Table 3 are largely irrelevant and only affect some relative peak values and not the overall behavior. Interestingly the character of the peaks is captured in a qualitative sense. Though the loading spectrum falls off sharply at $1500 \mathrm{~Hz}$, the nonlinear modal simulations of membrane displacement response using the $\mathrm{B}, \mathrm{BM}$, and UBC bases extend to twice that frequency because of period doubling. Membrane displacement response computed using the $\mathrm{CBC}$ basis does not exhibit period doubling.

The bending stress PSDs at the lowest excitation level are shown in Figure 9. All modal bases compare favorably with the results from numerical simulation in physical DoFs at this level. The membrane stress PSDs are shown in Figure 10. With regard to the bending only modal basis, the membrane stress arises from the bendingmembrane coupling in the stress recovery calculation, even though a zero membrane displacement is imposed. Results computed using the bending only and UBC bases over-predict the membrane stress at the first perioddoubled peak, and capture the behavior up to the third peak at about $370 \mathrm{~Hz}$. Above that frequency, the magnitudes differ significantly from the ABAQUS results. The same is true of the $\mathrm{CBC}$ basis, except that it additionally exhibits peaks at the bending frequencies. The $\mathrm{B}, \mathrm{UBC}$ and $\mathrm{CBC}$ membrane displacements are vastly different than that of the BM basis, yet the membrane stresses have similar characteristics. It thus appears that the membrane stress in the low frequency range is more dependent upon the bending displacement than on the membrane displacement. The BM results compare favorably to a frequency of about $700 \mathrm{~Hz}$, above which more significant differences are noted. This suggests that additional membrane modes in the BM basis are required to achieve membrane stress comparisons on a par with the membrane displacement comparisons. Above approximately 1150 $\mathrm{Hz}$, results from all modal bases give similar results, and differ significantly with those from numerical simulation in physical DoFs.

The following results and subsequent figures for the high loadings have been smoothed to more clearly observe the behavior. At the highest loading of $0.9216 \mathrm{lb} / \mathrm{in}$., the transverse displacement PSD exhibits the peak spreading and shifting characteristic of spring hardening nonlinearity, see Figure 11. For this case, the bending only basis indicates a higher degree of nonlinearity than the ABAQUS results, as evidenced by the first and second peaks shifted to higher frequencies, greater broadening, and smaller magnitudes. The UBC and CBC results are similar to the bending modes only, except that the fundamental mode is more accurately captured. The BM basis results compare well across the frequency range, with somewhat broader peaks than indicated by the numerical simulation in physical DoFs. The membrane behavior shown in Figure 12 follows the observations made at the lower level. The excellent agreement between BM basis and ABAQUS results demonstrate the efficacy of the BM basis in the highly nonlinear regime.

Bending and membrane stress PSDs are shown for the highest loading in Figure 13 and Figure 14, respectively. The BM basis most closely compares with the ABAQUS bending stress across the frequency range. The bending only, UBC and $\mathrm{CBC}$ bases significantly under-predict the bending stress of the first and second modes, and above about $1100 \mathrm{~Hz}$ indicate different character than the BM basis and ABAQUS results. Further, the UBC and CBC bases exhibit a non-physical twin peak in the vicinity of the first mode. In Figure 14, the BM basis results compare favorably with the ABAQUS results across the frequency range. The bending only, $\mathrm{UBC}$ and to a lesser extent $\mathrm{CBC}$ 
bases over-predict the membrane stress magnitude at the peak as well as the width of the peak. Above about 1100 $\mathrm{Hz}$, the character substantially differs from the ABAQUS results.

\section{Elevated Temperature}

The bending and membrane displacement and stress behavior for a beam under combined thermal and acoustic loading is next considered. The dimensions, material properties and boundary conditions used were the same as those previously used. A coefficient of thermal expansion of $1.24 \times 10^{-5} 1 /{ }^{\circ} \mathrm{F}$ was additionally specified. The thermal loading imposed was such that the beam remained in the pre-buckled regime.

The modes included in each modal basis were identical to those used for the room temperature case. Results computed using these "cold" modes compared more favorably with numerical simulation in physical DoFs than did those computed with "hot" modes. In the following, only results computed using the "cold" modes are presented.

The RMS transverse displacements are shown in Figure 15. Unlike the room temperature case, transverse displacements for the elevated temperature case indicate no clear winner across the loading range. Depending on the level of dynamic loading, results from a particular basis may compare more or less favorably than the others. The membrane displacements shown in Figure 16, however, indicate the best comparison with the BM basis. Like the room temperature case, the largest contribution to the RMS membrane displacement using the UBC comes from very small eigenvalues of the companion modes. At the lowest dynamic loading, the trends observed in the transverse displacement PSD (Figure 17) follow those from the room temperature case. Similarly, the membrane displacement PSD (Figure 18) shows good agreement only between the BM basis and ABAQUS, with the most significant discrepancy in magnitude at the first peak. The bending stress PSD (Figure 19) and membrane stress PSD (Figure 20) have similar trends to those observed for the room temperature case.

At the higher load level of $0.9216 \mathrm{lb} / \mathrm{in}$., the transverse displacement PSDs from all modal bases capture the fundamental mode fairly well, as shown in Figure 21. Only the BM basis describes the second mode behavior well, with the other bases indicating reduced amplitude and greater broadening. The membrane displacement PSD computed using the BM basis is the only one to compare well with the ABAQUS results, see Figure 22. The bending and membrane stress PSDs are shown in Figure 23 and Figure 24, respectively. Like the room temperature case, the bending stress results obtained using the BM basis are the most accurate, with the bending modes, UBC and $\mathrm{CBC}$ bases under-predicting the peaks. Further, the UBC and $\mathrm{CBC}$ bases again exhibit the non-physical double peak at the first mode. The low frequency membrane behavior is most accurately captured using the bending only basis, followed by the BM basis, which indicates greater peak broadening. The BM basis most accurately predicts the high frequency membrane behavior.

This set of results demonstrates the effect of modal basis selection on the nonlinear modal simulation of thermalacoustic response in the pre-buckled regime. The quality of displacement and stress PSDs are consistent with the room temperature case.

\section{Plate Results}

A simply supported aluminum plate was next considered to help understand the complexities of modal basis selection for a two-dimensional structure. The plate measured 10-in. $\mathrm{x} 14$-in. $\mathrm{x} 0.04$-in $(l \times w \times h)$ with the material properties indicated in Eqs. (4) and was subjected to a uniformly distributed acoustic load with a bandwidth of 1024 $\mathrm{Hz}$.

The MSC.NASTRAN model consisted of 560 CQUAD4 elements measuring 0.5 -in. x 0.5 -in. The ABAQUS model was similarly constructed using S4R elements. Nonlinear modal simulations were run using three modal bases; B, BM and UBC. For the bending modes only basis, the first five doubly symmetric bending modes (eigenvectors 1, 4, 8, 11, 12) were included. These occurred at natural frequencies of 58, 217, 373, 529 and $543 \mathrm{~Hz}$. For the BM basis, the first four anti-symmetric membrane modes (eigenvectors 276, 281, 364, 373) were additionally included for a total of 9 modes. These occurred at natural frequencies of 15.2, 15.5, $21.3 \mathrm{and} 22.1 \mathrm{kHz}$. The selection of these particular membrane modes was somewhat arbitrary as there was no guiding rationale. Their identification amongst the hundreds of eigenvectors was also difficult. For the UBC basis, the five companions of the bending modes were included for a total of 10 modes.

For this structure, an alternative nonlinear modal simulation using a direct stiffness evaluation method ${ }^{2}$ was also available. This approach neglects in-plane inertia and statically condenses the membrane displacement into the bending displacement. Since this approach requires manipulating the nonlinear stiffness formulation, it is not possible to implement in general purpose commercial codes. To avoid confusion, this nonlinear modal simulation will subsequently be referred to as "static physical condensation" method. A quarter-plate finite element model was constructed from 140 0.5-in. square modules, each having two MIN3-derived triangular elements with first order shear deformation. ${ }^{2}$ Symmetry conditions were imposed along the two centerlines. In the stress calculations to 
follow, the element stresses of the two triangular elements within the module were averaged to obtain the stress at the center of the square module. This allowed the stress from the static physical condensation method to be compared with the nonlinear modal simulations and simulations in physical DoFs.

Mass proportional damping corresponding to critical damping of $2.0 \%$ for the first bending mode was used in all three analyses. Displacement results are presented at the $(1 / 4,1 / 4)$ point at the coordinate position (2.5-in., 3.5-in.), and element stresses nearest the $(1 / 4,1 / 4)$ point at the coordinate position $(2.25$-in., 3.25 -in.). The stresses presented are along the short (10-in.) direction.

The transverse displacement PSDs for an acoustic loading of $106 \mathrm{~dB}$ are shown in Figure 25. All nonlinear modal simulations including the static physical condensation method agree well with the ABAQUS results. Differences in the peak amplitudes and frequencies, and the lack of higher frequency modes in the modal simulation are clearly attributable to lack of converged meshes and to modal truncation. The corresponding membrane displacements PSDs are shown in Figure 26. Results computed using the BM basis compare very well with the numerical simulation in physical DoFs through about half the frequency range, above which additional membrane modes in the basis appear to be required. Results from the static physical condensation capture the first perioddoubled peak, and attempt to capture the second and third period-doubled peaks. However, membrane displacements calculated using this method lack the essential character elsewhere. The UBC results indicate near zero membrane response, except at very low frequency. Figure 27 shows the bending stresses computed using each of the modal simulations compares favorably at the peaks, with differences elsewhere again attributable to lack of mesh convergence. Interestingly, the membrane stresses from the modal simulations agree fairly well with each other, and begin to differ substantially from the ABAQUS results above $300 \mathrm{~Hz}$, see Figure 28. Since both the bending only and UBC modal bases produce membrane stresses on a par with those from the BM basis and static physical condensation, it further substantiates the contention that the membrane stress is more highly dependent on the bending displacement than on the membrane displacement.

For an acoustic loading of $160 \mathrm{~dB}$, a high degree of nonlinearity is observed in the transverse displacement PSDs shown in Figure 29. What was the first resonant peak at $58 \mathrm{~Hz}$ has substantially broadened and shifted to about 300 Hz. Results from the BM basis and static physical condensation methods compare the best with numerical simulation in physical DoFs at the peak. The merits of basis selection or method used for the membrane displacement PSD is ambiguous, see Figure 30. At best, it can be seen that the UBC basis gives an effectively zero membrane response. The precise cause for this lack of agreement is presently unknown, although truncation of membrane modes in the BM basis is likely responsible for some of its difference. Finally, all of the modal simulations capture the peak bending stress response at about $300 \mathrm{~Hz}$, as shown in Figure 31. The off-peak behavior, particularly at the higher frequencies, substantially differs from the numerical simulation in physical DoFs. The membrane stress PSDs from all modal simulations agree fairly well across the frequency range and capture the period-doubled peak at around $600 \mathrm{~Hz}$, see Figure 32.

In general, results obtained from any of the modal simulations of the plate response are of lesser quality than the modal simulations of the beam response, relative to their respective numerical simulations in physical DoFs. Some differences are clearly identifiable through observation of the low response level, including modal truncation and inadequate mesh convergence. These are likely to have also affected the quality of results at the higher levels, but in a less transparent way. In addition, since the membrane modes for the plate are more complicated than those of the beam, e.g. two-dimensional versus one-dimensional, the likelihood of selecting the proper modes for inclusion in the $\mathrm{BM}$ basis is lessened. Thus, the advantages of using the BM basis are diminished by practical considerations that may be difficult to overcome.

\section{Conclusions}

The effect of modal basis selection on the displacement and stress response predicted using a nonlinear modal simulation was investigated. For the beam, it was found that

- the bending and membrane modal basis offered the only accurate prediction of membrane displacement and the best prediction of bending and membrane stress,

- the bending modes only basis offered the second best approach as it did not produce anomalous behavior in the displacement or stress PSDs, did not increase the size of the coupled set of modal equations, and did not have the added complication of identifying membrane modes or computing companion modes,

- either variant of the companion modes basis ( $\mathrm{CBC}$ or UBC) produced non-physical behavior in the displacement and stress PSDs, and therefore their use is not recommended, 
- there is no advantage for computing companion modes dynamically (as presented in this paper) versus statically, ${ }^{7,9}$ nor is there an engineering rationale for selection of eigenvector (for dynamic) or prescribed displacement or loading (for static),

- there is no established criteria for selection of which modes to include in the basis for any of the membrane variants (BM, UBC, $\mathrm{CBC})$.

Results for the beam demonstrated that it is possible to construct an effective bending and membrane modal basis through the use of engineering judgment and some effort to identify the particular membrane modes selected. For the plate, it was additionally found that

- the selection and identification of modes was more difficult and the accuracy of the predictions suffered,

- the static physical condensation approach was able to accurately predict the first mode of the membrane displacement. It has the advantages of not increasing the system size and not having to identify or calculate membrane or companion modes, but is limited to implementation in special purpose codes.

In general, increasing structural complexity requires additional bending and membrane modes in the basis to obtain the same level of accuracy.

Finally, for the problems considered (those with nonlinear bending-membrane coupling), the calculation of membrane stress was more dependent upon the bending displacement than on the membrane displacement, particularly at low frequencies. Two areas for future study include the effect of basis selection on fatigue life predictions, and the effect of basis selection for problems that exhibit other types of nonlinearity, e.g. large rotation for cantilevered structures.

\section{References}

${ }^{1}$ Mei, C., Dhainaut, J.M., Duan, B., Spottswood, S.M., and Wolfe, H.F., "Nonlinear random response of composite panels in an elevated thermal environment," Air Force Research Laboratory AFRL-VA-WP-TR-2000-3049, Wright-Patterson Air Force Base, $\mathrm{OH}$, October 2000.

${ }^{2}$ Przekop, A., "Nonlinear response and fatigue estimation of aerospace curved surface panels to combined acoustic and thermal loads," Ph.D. Dissertation, Old Dominion University, Norfolk, VA, 2003.

${ }^{3}$ Przekop, A., Guo, X., Azzouz, M.S., and Mei, C., "Reinvestigation of nonlinear random response of shallow shells using finite element modal formulation," Proceedings of the 45th AIAA/ASME/ASCE/AHS/ASC Structures, Structural Dynamics and Materials Conference, AIAA-2004-1553, Palm Springs, CA, 2004.

${ }^{4}$ McEwan, M.I., Wright, J.R., Cooper, J.E., and Leung, Y.T., "A finite element/modal technique for nonlinear plate and stiffened panel response prediction," Proceedings of the 42nd AIAA/ASME/ASCE/AHS/ASC Structures, Structural Dynamics, and Materials Conference, AIAA-2001-1595, Seattle, WA, 2001.

${ }^{5}$ Muravyov, A.A. and Rizzi, S.A., "Determination of nonlinear stiffness with application to random vibration of geometrically nonlinear structures," Computers and Structures, Vol. 81, No. 15, 2003, pp. 1513-1523.

${ }^{6}$ Rizzi, S.A. and Muravyov, A.A., "Improved equivalent linearization implementations using nonlinear stiffness evaluation," NASA TM-2001-210838, March 2001.

${ }^{7}$ Hollkamp, J.J., Gordon, R.W., and Spottswood, S.M., "Nonlinear sonic fatigue response prediction from finite element modal models: a comparison with experiments," Proceedings of the 44th AIAA/ASME/ASCE/AHS/ASC Structures, Structural Dynamics, and Materials Conference, AIAA-2003-1709, Norfolk, VA, 2003.

${ }^{8}$ Rizzi, S.A. and Muravyov, A.A., "Comparison of nonlinear random response using equivalent linearization and numerical simulation," Structural Dynamics: Recent Advances, Proceedings of the 7th International Conference, Vol. 2, Southampton, UK, 2000, N.S. Ferguson, et al. (ed.), pp. 833-846.

${ }^{9}$ Mignolet, M.P., Radu, A.G., and Gao, X., "Validation of reduced order modeling for the prediction of the response and fatigue life of panels subjected to thermo-acoustic effects," Structural Dynamics: Recent Advances, Proceedings of the 8th International Conference, Southampton, UK, 2003, M.J. Brennan, et al. (ed.).

${ }^{10}$ Press, W.H., Teukolsky, S.A., Vetterling, W.T., and Flannery, B.P., "Numerical recipes, the art of scientific computing," CDROM v2.10, Cambridge University Press, 2002. 
Table 1: Diagonal terms of linear stiffness obtained using five different modal bases. Shaded cells indicate bending eigenvalues.

\begin{tabular}{|c|c|c|c|c|c|}
\hline & B & BM & CBC & UBC & $\mathrm{UBC}^{9}$ (static) \\
\hline$d_{1}^{1}$ & $1.318 \mathrm{E}+05$ & $1.318 \mathrm{E}+05$ & $1.318 \mathrm{E}+05$ & $1.318 \mathrm{E}+05$ & $1.318 \mathrm{E}+05$ \\
\hline$d_{2}^{2}$ & $3.845 \mathrm{E}+06$ & $3.845 \mathrm{E}+06$ & $3.845 \mathrm{E}+06$ & $3.845 \mathrm{E}+06$ & $3.845 \mathrm{E}+06$ \\
\hline$d_{3}^{3}$ & $2.341 \mathrm{E}+07$ & $2.341 \mathrm{E}+07$ & $2.341 \mathrm{E}+07$ & $2.341 \mathrm{E}+07$ & $2.341 \mathrm{E}+07$ \\
\hline$d_{4}^{4}$ & $8.079 \mathrm{E}+07$ & $8.079 \mathrm{E}+07$ & $8.080 \mathrm{E}+07$ & $8.079 \mathrm{E}+07$ & $8.079 \mathrm{E}+07$ \\
\hline$d_{5}^{5}$ & N/A & $4.988 \mathrm{E}+09$ & N/A & $6.607 \mathrm{E}+00$ & $2.168 \mathrm{E}+02$ \\
\hline$d_{6}^{6}$ & N/A & $1.991 \mathrm{E}+10$ & N/A & $1.029 \mathrm{E}+02$ & $8.083 \mathrm{E}+01$ \\
\hline$d_{7}^{7}$ & N/A & $4.467 \mathrm{E}+10$ & N/A & $3.208 \mathrm{E}+02$ & $7.326 \mathrm{E}+02$ \\
\hline$d_{8}^{8}$ & N/A & $7.908 \mathrm{E}+10$ & N/A & $6.195 \mathrm{E}+02$ & $3.210 \mathrm{E}+02$ \\
\hline
\end{tabular}

Table 2: Diagonal terms of cubic stiffness obtained using five different modal bases.

\begin{tabular}{|c|c|c|c|c|c|}
\hline & $\mathrm{B}$ & $\mathrm{BM}$ & $\mathrm{CBC}$ & $\mathrm{UBC}$ & $\mathrm{UBC}^{9}\left(\mathrm{static}^{\prime}\right.$ \\
\hline$b_{111}^{1} q_{1}^{2}$ & $5.062 \mathrm{E}+02$ & $5.062 \mathrm{E}+02$ & $5.062 \mathrm{E}+02$ & $5.062 \mathrm{E}+02$ & $5.062 \mathrm{E}+02$ \\
\hline$b_{222}^{2} q_{2}^{2}$ & $3.615 \mathrm{E}+04$ & $3.615 \mathrm{E}+04$ & $3.615 \mathrm{E}+04$ & $3.615 \mathrm{E}+04$ & $3.615 \mathrm{E}+04$ \\
\hline$b_{333}^{3} q_{3}^{2}$ & $2.488 \mathrm{E}+05$ & $2.488 \mathrm{E}+05$ & $2.488 \mathrm{E}+05$ & $2.488 \mathrm{E}+05$ & $2.488 \mathrm{E}+05$ \\
\hline$b_{444}^{4} q_{4}^{2}$ & $8.998 \mathrm{E}+05$ & $8.998 \mathrm{E}+05$ & $8.998 \mathrm{E}+05$ & $8.998 \mathrm{E}+05$ & $8.998 \mathrm{E}+05$ \\
\hline$b_{555}^{5} q_{5}^{2}$ & N/A & $9.257 \mathrm{E}-05$ & N/A & $-2.622 \mathrm{E}-08$ & $4.667 \mathrm{E}-07$ \\
\hline$b_{666}^{6} q_{6}^{2}$ & N/A & $1.022 \mathrm{E}-03$ & N/A & $5.324 \mathrm{E}-08$ & $5.568 \mathrm{E}-05$ \\
\hline$b_{777}^{7} q_{7}^{2}$ & N/A & $-6.789 \mathrm{E}-04$ & N/A & $-1.642 \mathrm{E}-07$ & $-5.252 \mathrm{E}-04$ \\
\hline$b_{888}^{8} q_{8}^{2}$ & N/A & $4.139 \mathrm{E}-03$ & N/A & $2.653 \mathrm{E}-07$ & $-4.691 \mathrm{E}-04$ \\
\hline
\end{tabular}

Table 3: Selected off-diagonal terms of cubic stiffness obtained using five different modal bases.

\begin{tabular}{|c|c|c|c|c|c|}
\hline & $\mathrm{B}$ & $\mathrm{BM}$ & $\mathrm{CBC}$ & $\mathrm{UBC}$ & $\mathrm{UBC}^{9}$ (static) \\
\hline$b_{111}^{1} q_{1}^{2}$ & $5.062 \mathrm{E}+02$ & $5.062 \mathrm{E}+02$ & $5.062 \mathrm{E}+02$ & $5.062 \mathrm{E}+02$ & $5.062 \mathrm{E}+02$ \\
\hline$b_{115}^{1} q_{1} q_{5}$ & $\mathrm{~N} / \mathrm{A}$ & $1.270 \mathrm{E}-06$ & $\mathrm{~N} / \mathrm{A}$ & $2.274 \mathrm{E}-11$ & $-8.560 \mathrm{E}-12$ \\
\hline$b_{155}^{1} q_{1} q_{5}$ & $\mathrm{~N} / \mathrm{A}$ & $\mathbf{- 1 . 1 7 3 E}+\mathbf{0 3}$ & $\mathrm{N} / \mathrm{A}$ & $-2.801 \mathrm{E}-06$ & $-6.428 \mathrm{E}-07$ \\
\hline$b_{555}^{1} q_{5}^{2}$ & $\mathrm{~N} / \mathrm{A}$ & $2.592 \mathrm{E}-06$ & $\mathrm{~N} / \mathrm{A}$ & $4.320 \mathrm{E}-14$ & $-1.638 \mathrm{E}-14$ \\
\hline$b_{111}^{5} q_{1}^{2}$ & $\mathrm{~N} / \mathrm{A}$ & $-8.380 \mathrm{E}-02$ & N/A & $1.730 \mathrm{E}-05$ & $2.610 \mathrm{E}-07$ \\
\hline$b_{115}^{5} q_{1} q_{5}$ & N/A & $\mathbf{- 1 . 0 6 6 E}+\mathbf{0 3}$ & N/A & $-2.557 \mathrm{E}-06$ & $-7.970 \mathrm{E}-07$ \\
\hline$b_{155}^{5} q_{1} q_{5}$ & N/A & $4.680 \mathrm{E}-01$ & N/A & $-5.137 \mathrm{E}-09$ & $9.984 \mathrm{E}-08$ \\
\hline$b_{555}^{5} q_{5}^{2}$ & N/A & $9.257 \mathrm{E}-05$ & N/A & $-2.622 \mathrm{E}-08$ & $4.667 \mathrm{E}-07$ \\
\hline
\end{tabular}




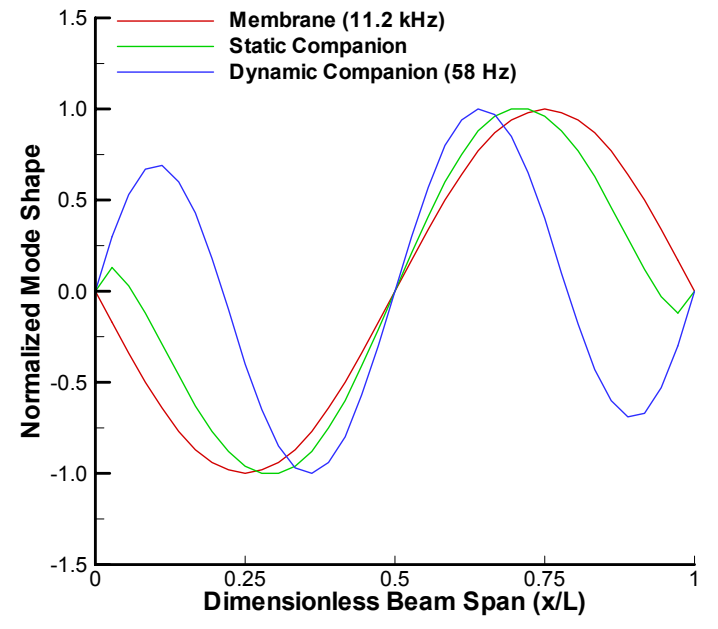

Figure 1: First anti-symmetric membrane and companion modes.

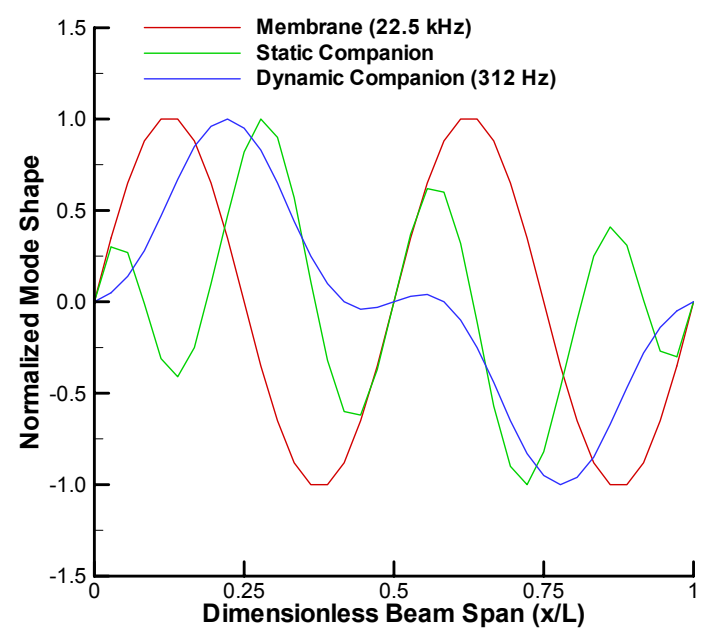

Figure 2: Second anti-symmetric membrane and companion modes.

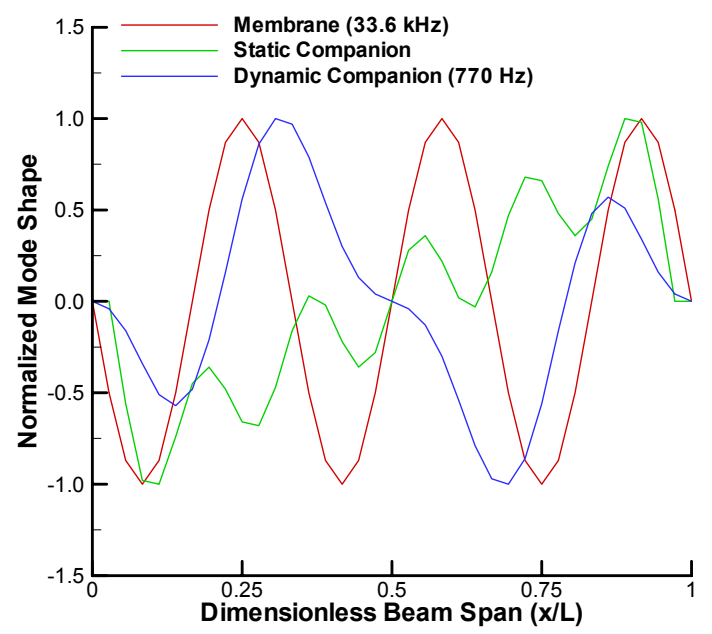

Figure 3: Third anti-symmetric membrane and companion modes.

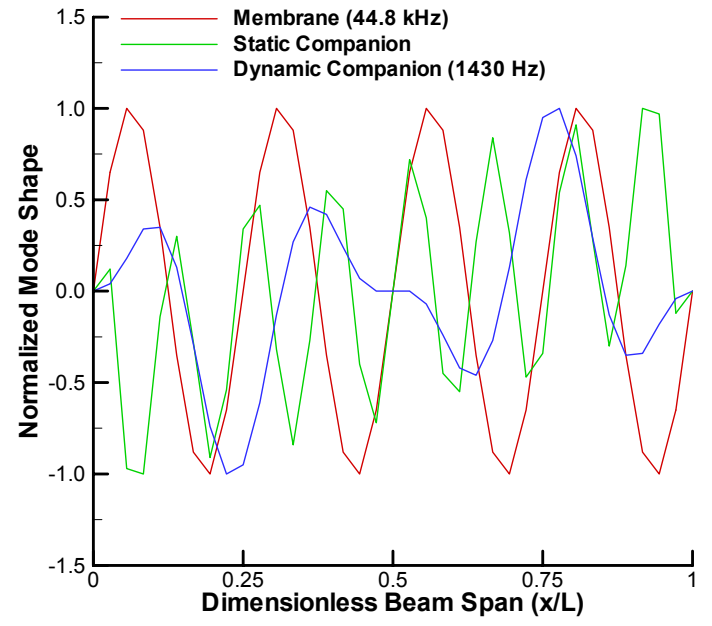

Figure 4: Fourth anti-symmetric membrane and companion modes.

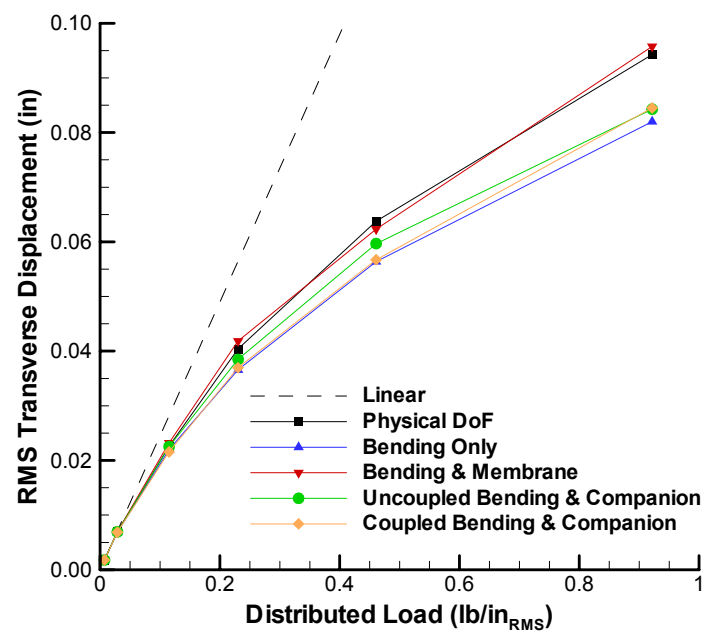

Figure 5: RMS transverse displacement as a function of distributed load.

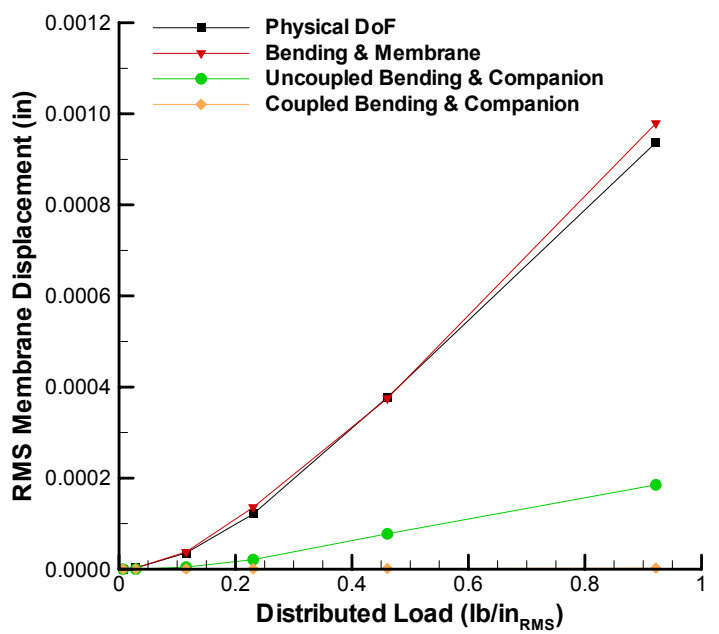

Figure 6: RMS membrane displacement as a function of distributed load. 


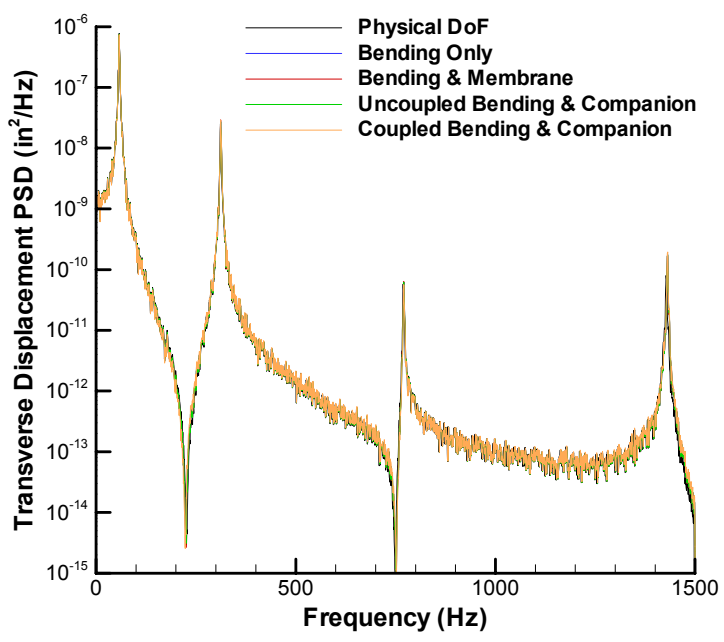

Figure 7: Transverse displacement PSD for $7.2 \times 10^{-3} \mathrm{lb} /$ in distributed load.

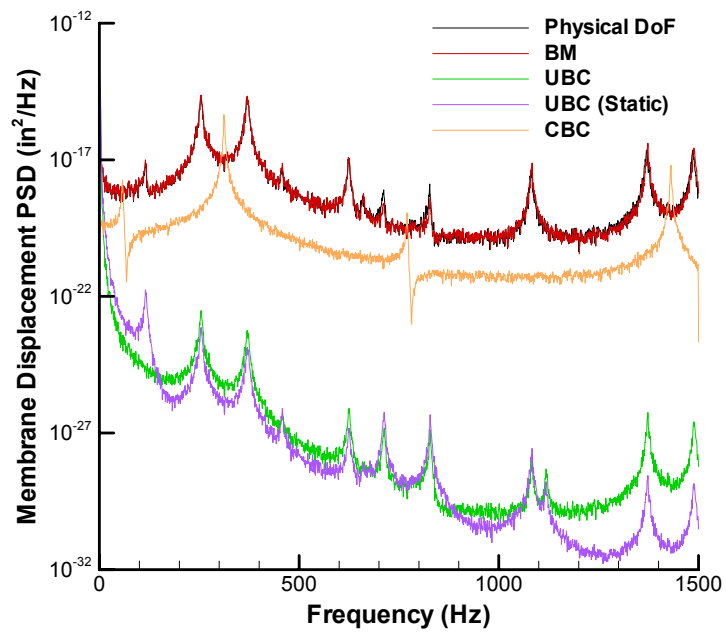

Figure 8: Membrane displacement PSD for $7.2 \times 10^{-3} \mathrm{lb} / \mathrm{in}$ distributed load.

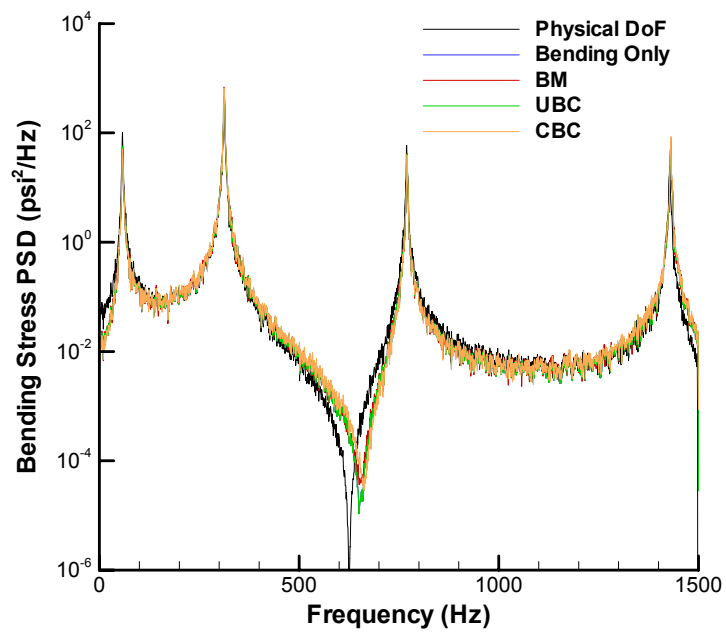

Figure 9: Bending stress PSD for $7.2 \times 10^{-3} \mathrm{lb} / \mathrm{in}$ distributed load.

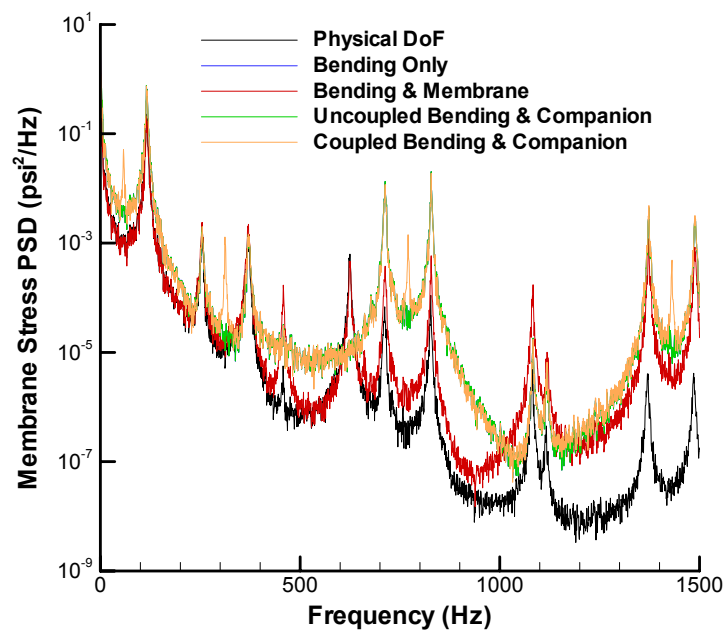

Figure 10: Membrane stress PSD for $7.2 \times 10^{-3} \mathrm{lb} /$ in distributed load

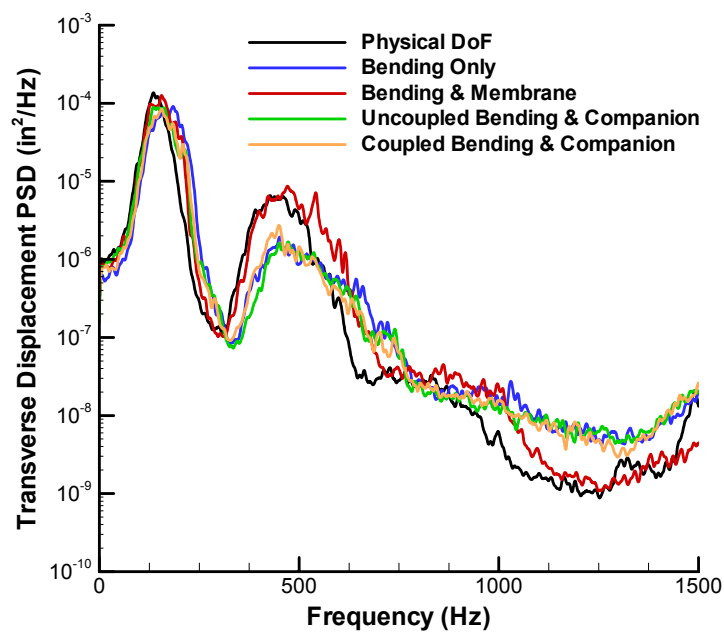

Figure 11: Transverse displacement PSD for $0.9216 \mathrm{lb} / \mathrm{in}$ distributed load.

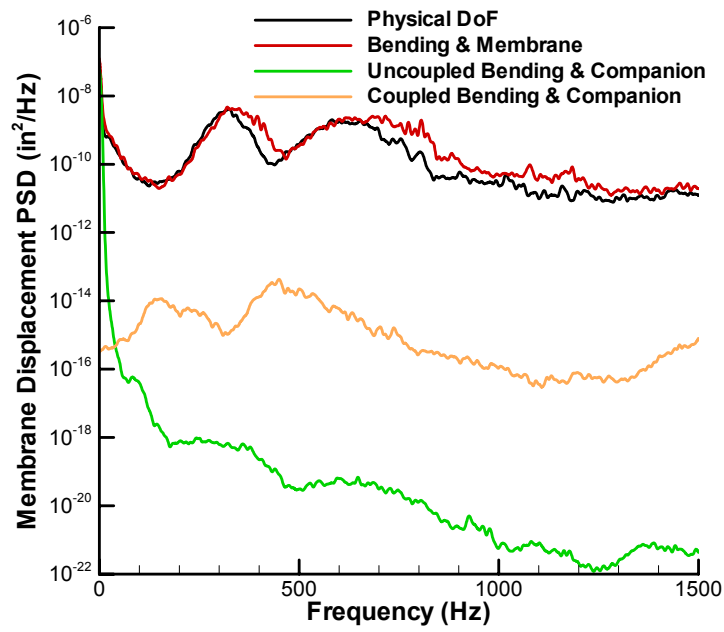

Figure 12: Membrane displacement PSD for $0.9216 \mathrm{lb} /$ in distributed load. 


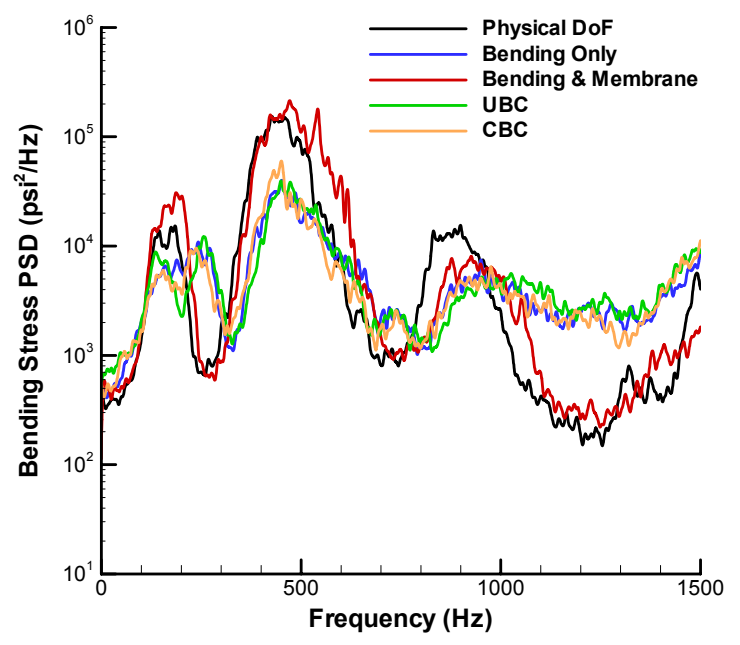

Figure 13: Bending stress PSD for $0.9216 \mathrm{lb} /$ in distributed load.

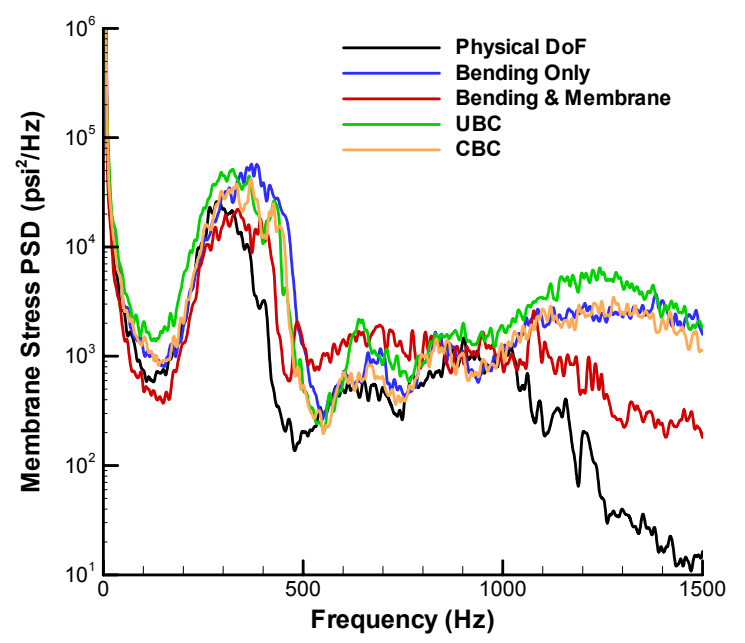

Figure 14: Membrane stress PSD for $0.9216 \mathrm{lb} /$ in distributed load.

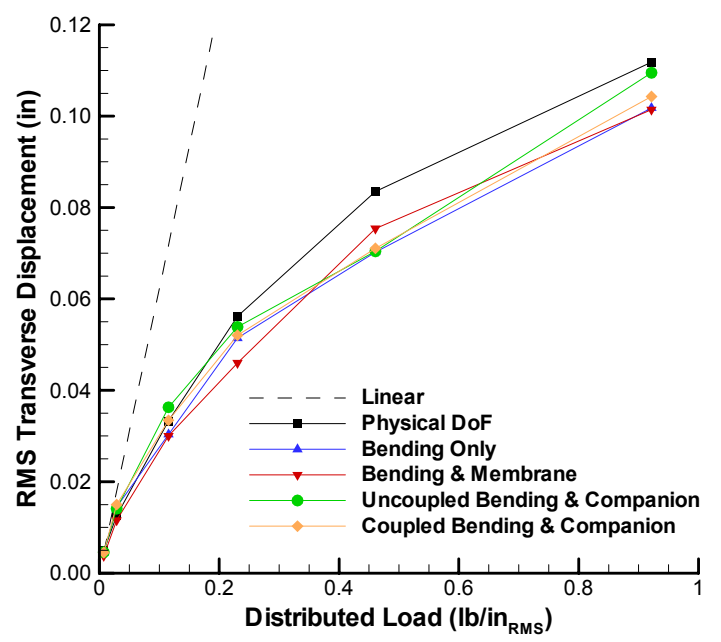

Figure 15: RMS transverse displacement for thermally pre-buckled beam.

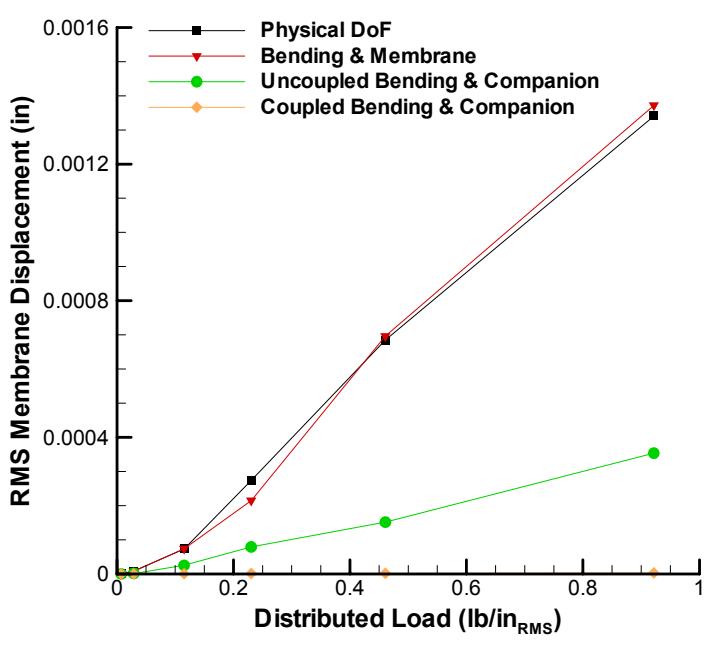

Figure 16: RMS membrane displacement for thermally pre-buckled beam.

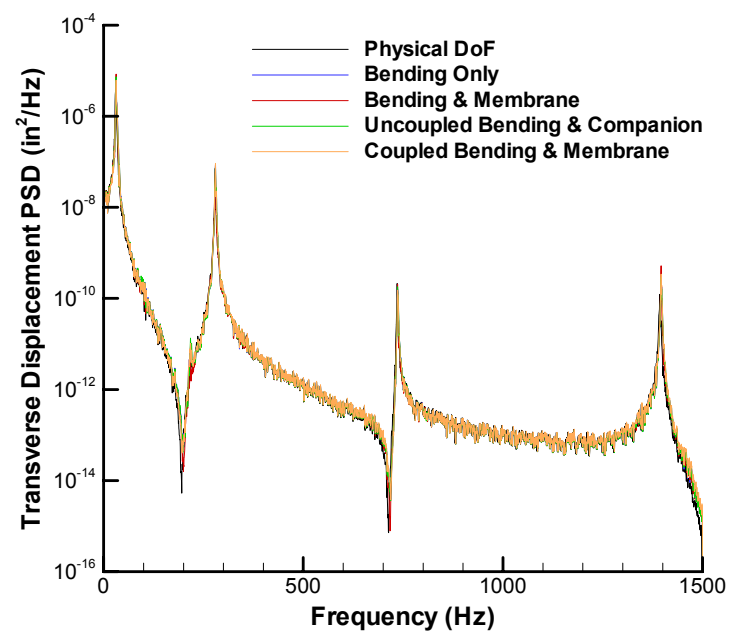

Figure 17: Transverse disp. PSD for thermally prebuckled beam $\left(7.2 \times 10^{-3} \mathrm{lb} /\right.$ in distributed load $)$.

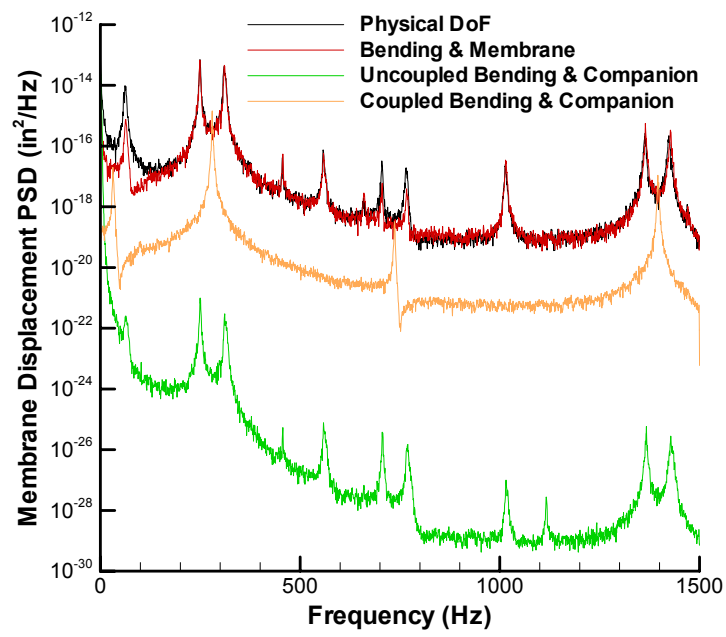

Figure 18: Membrane disp. PSD for thermally prebuckled beam $\left(7.2 \times 10^{-3} \mathrm{lb} /\right.$ in distributed load). 


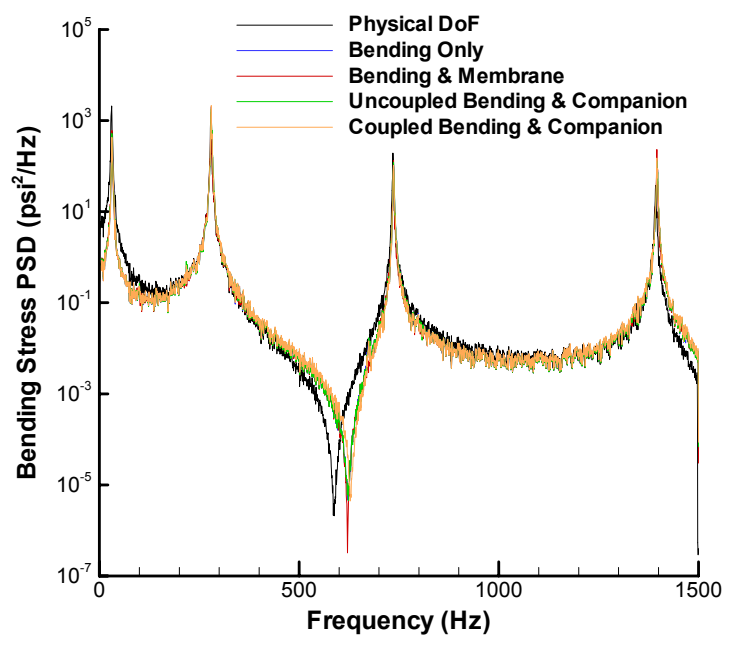

Figure 19: Bending stress PSD for thermally prebuckled beam $\left(7.2 \times 10^{-3} \mathrm{lb} /\right.$ in distributed load).

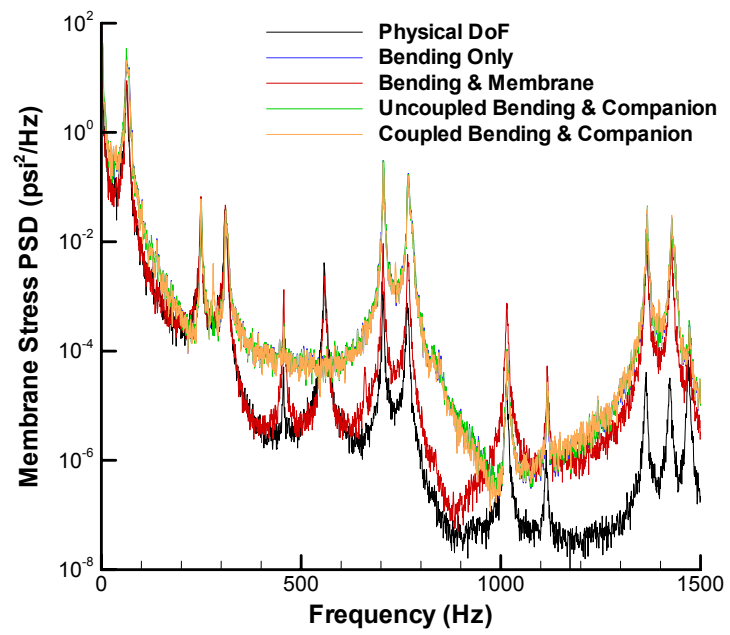

Figure 20: Membrane stress PSD for thermally prebuckled beam $\left(7.2 \times 10^{-3} \mathrm{lb} /\right.$ in distributed load $)$.

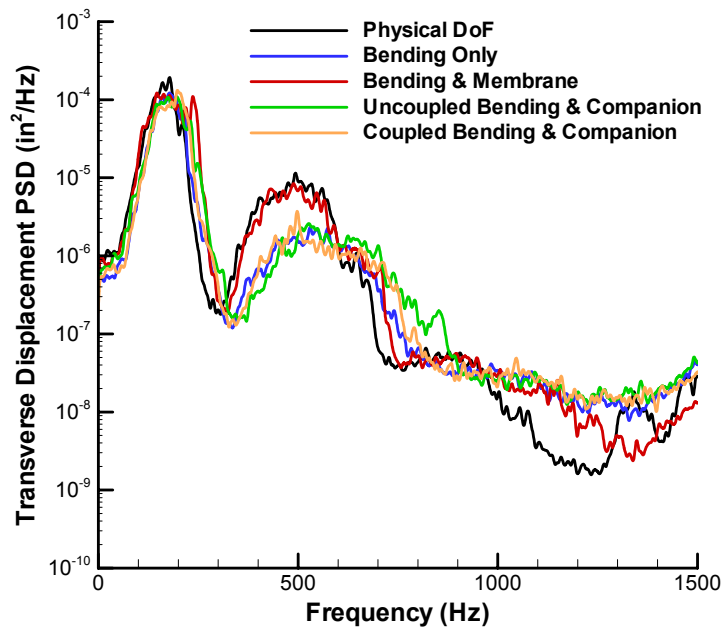

Figure 21: Transverse disp. PSD for thermally prebuckled beam (0.9216 lb/in distributed load).

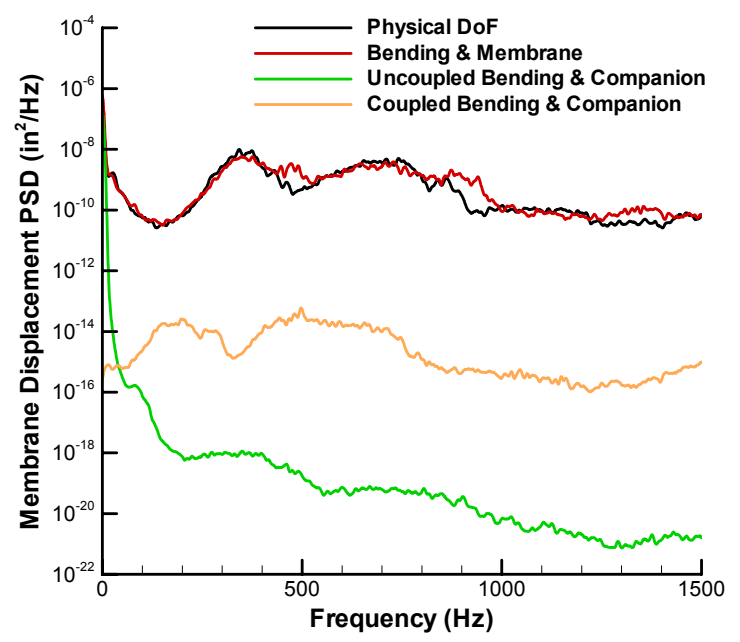

Figure 22: Membrane disp. PSD for thermally prebuckled beam (0.9216 lb/in distributed load).

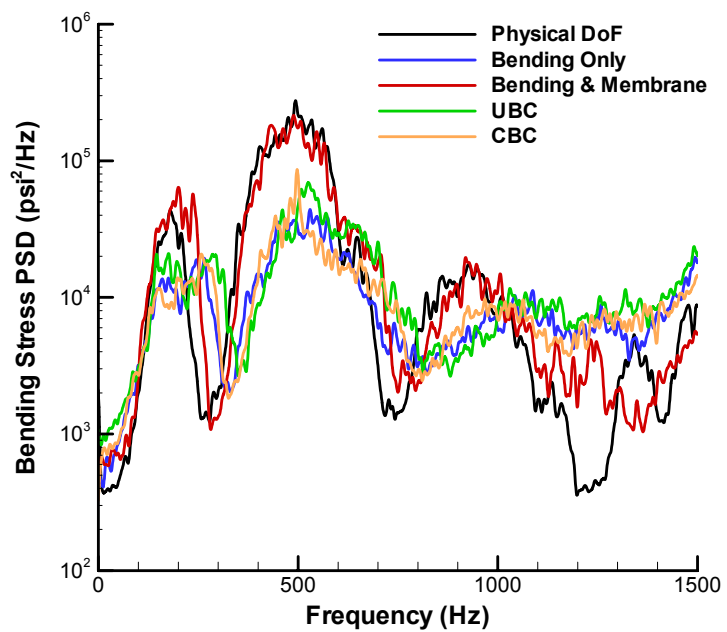

Figure 23: Bending stress PSD for thermally prebuckled beam (0.9216 lb/in distributed load).

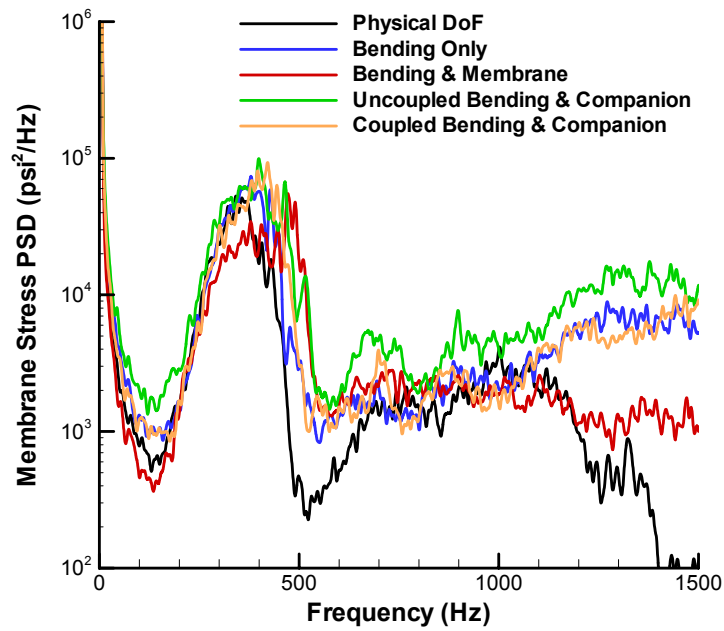

Figure 24: Membrane stress PSD for thermally prebuckled beam (0.9216 lb/in distributed load). 


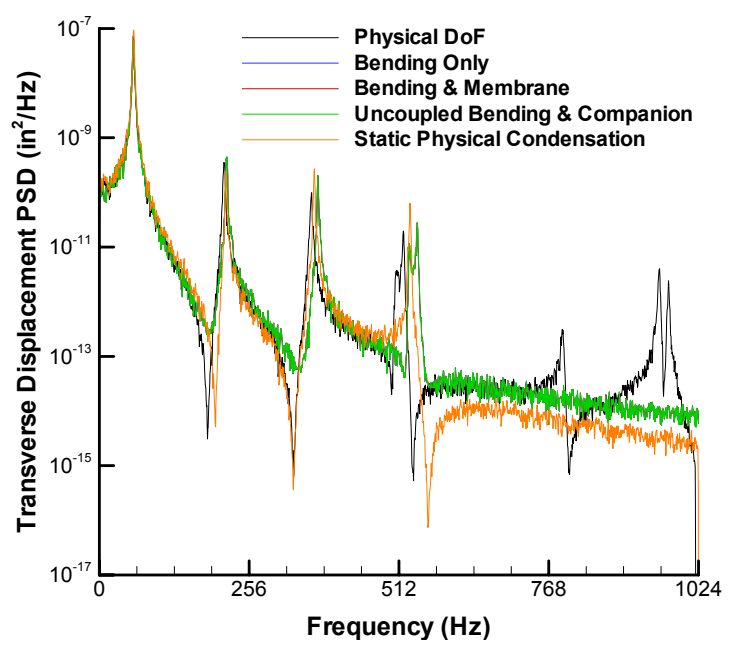

Figure 25: Transverse displacement PSD for plate subjected to $106 \mathrm{~dB}$ acoustic loading.

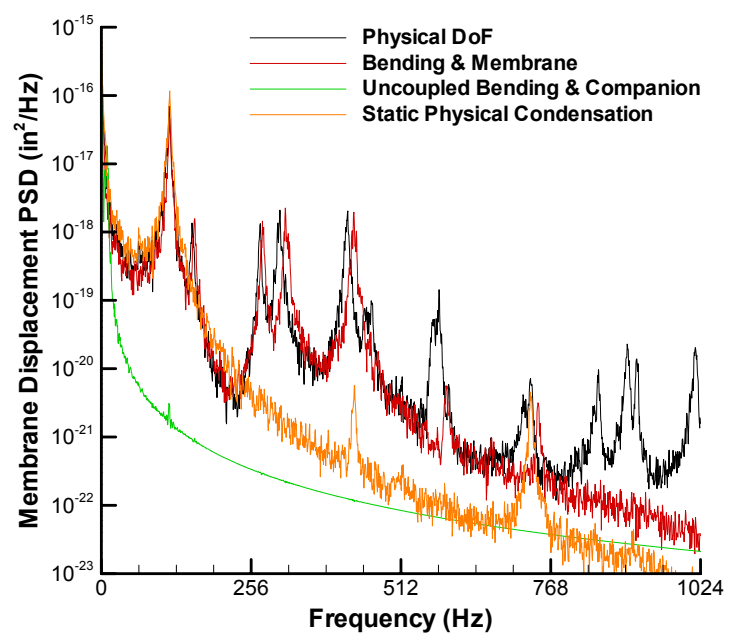

Figure 26: Membrane displacement PSD for plate subjected to $106 \mathrm{~dB}$ acoustic loading.

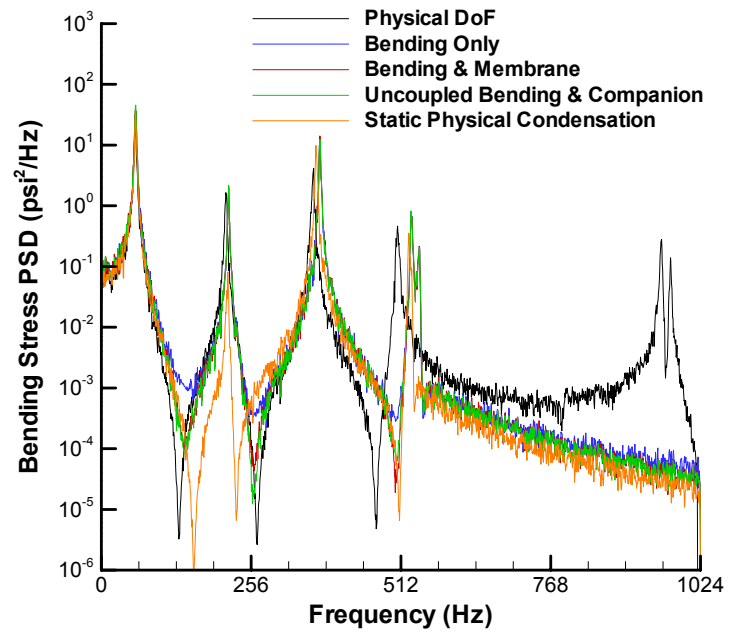

Figure 27: Bending stress PSD for plate subjected to $106 \mathrm{~dB}$ acoustic loading.

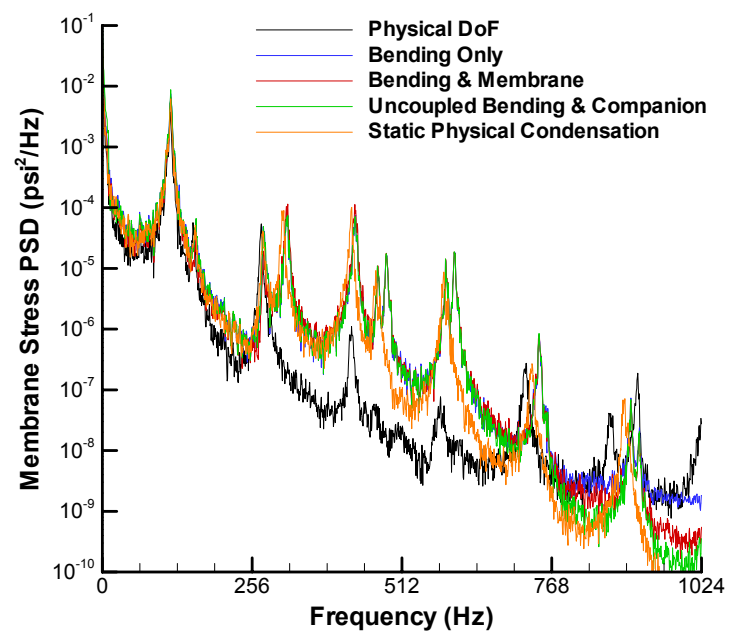

Figure 28: Membrane stress PSD for plate subjected to $106 \mathrm{~dB}$ acoustic loading.

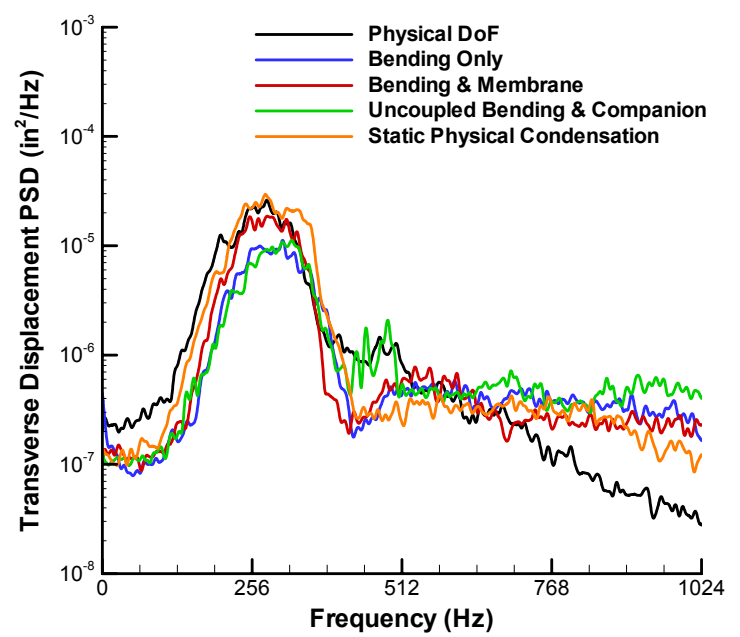

Figure 29: Transverse displacement PSD for plate subjected to $160 \mathrm{~dB}$ acoustic loading.

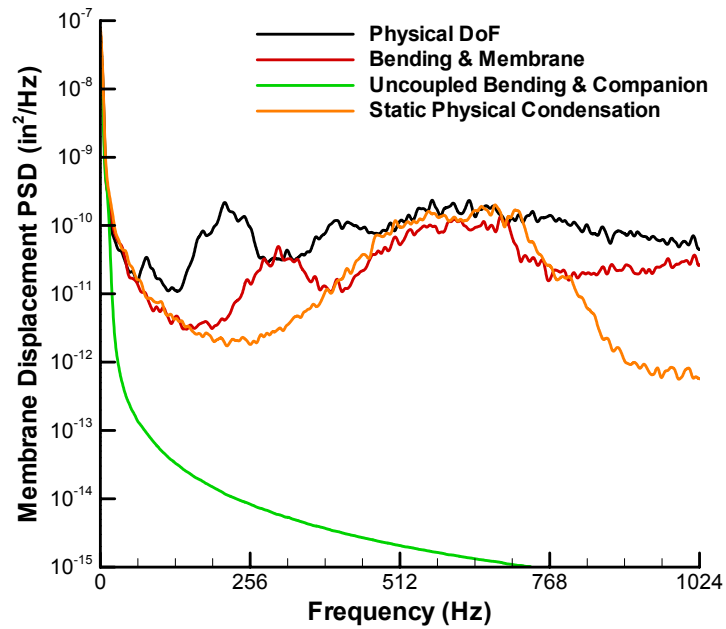

Figure 30: Membrane displacement PSD for plate subjected to $160 \mathrm{~dB}$ acoustic loading. 


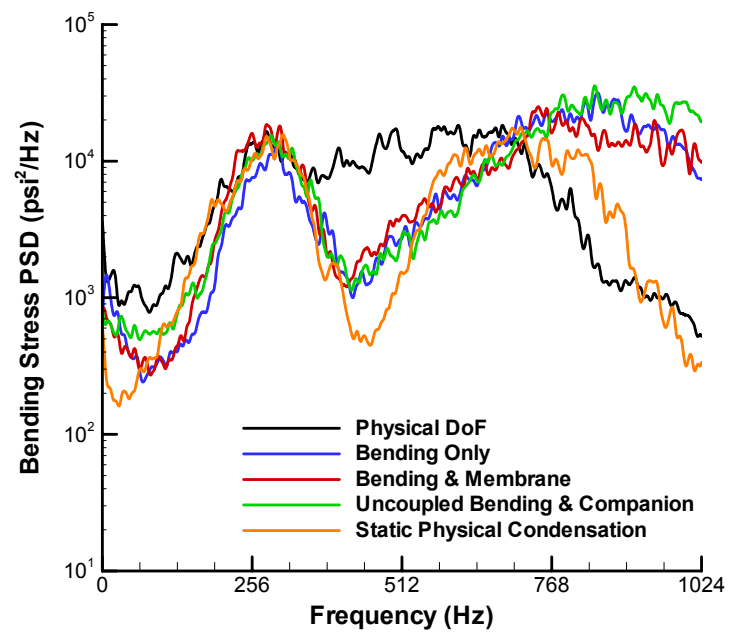

Figure 31: Bending stress PSD for plate subjected to $160 \mathrm{~dB}$ acoustic loading.

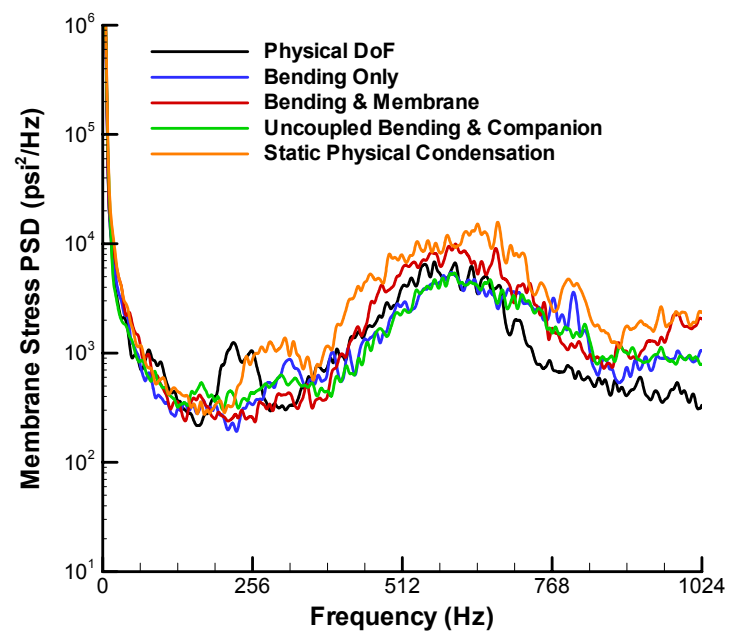

Figure 32: Membrane stress PSD for plate subjected to $160 \mathrm{~dB}$ acoustic loading. 